\title{
CONCERNING THE FINITE MODEL PROPERTY FOR PROPOSITIONAL CALCULI
}

\author{
JOHN ANDERSON
}

Introduction. For the definitions of propositional calculi, models and the finite model property (f.m.p.) we refer to [1] (we use 'model' for 'strong model').

In this note we describe two equivalent propositional calculi (equivalent in that they have identical sets of theorems) which differ in that one has the f.m.p. and the other does not, and in fact has no nontrivial finite model at all. This shows the f.m.p. is an attribute of a propositional calculus and not of the equivalence class of the calculus, unlike, for instance, decidability.

I should like to thank R. Harrop for conversations on the topics of this paper, and also thank the referee for suggesting considerable improvements.

A propositional calculus without the f.m.p. Consider the propositional calculus $P$ which has the single binary connective $\rightarrow$, modus ponens with respect to $\rightarrow$, and axiom schemes

I. $X \rightarrow X$,

II. $(I X \rightarrow X) \rightarrow Y$,

III. $((I X \rightarrow Y) \rightarrow X) \rightarrow\left(\left(I^{2} X \rightarrow Y\right) \rightarrow Z\right)$,

where $X, Y$ and $Z$ stand for arbitrary formulae and $I X$ is an abbreviation for $X \rightarrow X, I^{2} X$ for $I(I X)$, etc.

Lemma 1. A formula is a theorem of $P$ if and only if it is of one of the forms:

(i) an instance of an axiom scheme or

(ii) $\left(I^{k} X \rightarrow X\right) \rightarrow Y$, where $k \geqq 1$.

Proof. Sufficiency is straightforward; (i) is obvious and (ii) follows by induction on $k$. For $k=1,\left(I^{k} X \rightarrow X\right) \rightarrow Y$ is axiom scheme II. If we assume the result for $k-1$ we have that

$$
\left(I^{k} X \rightarrow X\right) \rightarrow I^{k-1} X \text { is a provable scheme. }
$$

From axiom scheme III we have

$$
\left(\left(I^{k} X \rightarrow X\right) \rightarrow\left(I^{k-1} X\right)\right) \rightarrow\left(\left(I^{k+1} X \rightarrow X\right) \rightarrow Z\right),
$$

and by an application of modus ponens we prove

Received by the editors May 15, 1967. 


$$
\left(I^{k+1} X \rightarrow X\right) \rightarrow Z .
$$

Necessity follows by induction on the length of proof of a theorem of $P$. A theorem with a proof of length one is an instance of an axiom scheme and is of form (i). A theorem $B$, with longer proof, if it is not of form (i) follows by modus ponens from two theorems $A, A \rightarrow B$, each of shorter proof than $B$, and so by the induction hypothesis satisfying (i) or (ii).

If $A \rightarrow B$ is an instance of $\mathrm{I}$, then $A=B$ and $B$ is of form (i) or (ii) because $A$ is. By inspection no instance of $\left(I^{k} X \rightarrow X\right)$, where $k \geqq 1$, can be of form (i) or (ii), and so $A \rightarrow B$ cannot be an instance of axiom scheme II or of form (ii). Thus $A \rightarrow B$ is an instance of axiom scheme III, and $A$ an instance of $(I X \rightarrow Y) \rightarrow X$. Hence $A$ cannot be a substituted case of axiom schemes I or III, which are specially designed to avoid this, and so, being of form (i) or (ii), must be an instance of a scheme

$$
\left(I^{k} X \rightarrow X\right) \rightarrow Y, \quad \text { where } k \geqq 1,
$$

and immediately we see that $B$ must be of form (ii).

A corollary of this lemma is that $P$ is consistent, that is not every formula is a theorem, and also that $P$ is decidable.

Lemma 2. Any nontrivial model of a propositional calculus in which modus ponens is satisfied and in which the schemes

$$
\begin{aligned}
& A_{0}: \quad X \rightarrow X \\
& A_{1}: \quad(I X \rightarrow X) \rightarrow Y \\
& A_{2}: \quad\left(I^{2} X \rightarrow X\right) \rightarrow Y \\
& A_{n-1}:\left(I^{n-1} X \rightarrow X\right) \rightarrow Y
\end{aligned}
$$

are valid has at least $\boldsymbol{n}$ distinct designated elements.

Proof. We adapt a technique from [1]. Let the model have a set $E$ of elements, and a set $D \subset E$ of designated elements. $D \neq E$ since the model is nontrivial. We note the following properties. If $a, b \in E$ and $a,(a \rightarrow b) \in D$, then $b \in D$ because the model satisfies modus ponens. Also $\left(I^{k} a \rightarrow a\right)$, where $1 \leqq k<n$, is undesignated for all $a \in E$, for if $\left(I^{k} a \rightarrow a\right) \in D$ for any $a \in E$, we could choose a nondesignated $b \in E$, and $\left(\left(I^{k} a \rightarrow a\right) \rightarrow b\right)$ would be undesignated, contradicting the validity of the scheme $A_{k}$. 
We have that $I^{n} a$ is designated for all $n \geqq 1$ and $a \in E$ since the model satisfies the scheme $A_{0}$. Thus, for $d \in D, d, I d, \cdots, I^{n-1} d$ are all designated. To complete the proof we show they are all distinct. Assuming the contrary, that is $I^{i} d=I^{j} d$ for some $i, j$, where $0 \leqq i<j<n$, we have $\left(I^{j} d \rightarrow I^{i} d\right)=\left(I^{j-i}\left(I^{i} d\right) \rightarrow I^{i} d\right)$ is designated, which contradicts what has been noted above.

THEOREM 1. The propositional calculus $P$ has no finite nontrivial model, and does not have the finite model property.

Proof. Any model of $P$ must satisfy modus ponens and make the scheme $\left(I^{k} X \rightarrow X\right) \rightarrow Y$ valid for every $k>1$, since any such instance is a theorem by Lemma 1 . But then, from Lemma 2 , the model needs an infinite set of designated elements.

Decidable propositional calculi with no finite models have previously been exhibited by R. Harrop (see, for example, [1]).

We remark in passing that the existence of a consistent propositional calculus with no nontrivial finite model can be deduced from the fact that there is no effective test to determine of an arbitrary propositional calculus whether or not it is consistent (see [2]); since no inconsistent calculus has a nontrivial model and if a finite nontrivial model exists for a calculus it can be effectively found.

A propositional calculus with the f.m.p. equivalent to $P$. Let $P^{*}$ be a propositional calculus with the same axiom schemes as $P$, but with the single rule

$$
\frac{(I X \rightarrow Y) \rightarrow X}{\left(I^{2} X \rightarrow Y\right) \rightarrow Z}
$$

The conclusion of this rule is the same as the conclusion of modus ponens with $(I X \rightarrow Y) \rightarrow X$ first premise, and axiom scheme III second premise. A similar proof to that of Lemma 1 establishes that the theorems of $P^{*}$ are just those formulae satisfying (i) or (ii), so that $P$ and $P^{*}$ are equivalent.

TheOREM 2. $P^{*}$ has the finite model property.

Proof. Define the length of a formula to be the number of symbols (parentheses, propositional variables and connectives) of which it consists.

Let $f$ be a formula of $P^{*}$ which is not a theorem, and let $\left\{s_{1}, \cdots, s_{n}\right\}$ be the finite set of all formulae constructable from the propositional variables of $f$ and of length less than or equal to the length of $f$. 
We construct a finite model which rejects $f$. The elements of this model are $\left\{s_{1}, \cdots, s_{n}, 1\right\}$. The designated elements are the provable formulae of $s_{1}, \cdots, s_{n}$ together with 1 . The truth table is defined by

$$
\begin{aligned}
(1 \rightarrow 1) & =1, \\
\left(1 \rightarrow s_{i}\right) & =1, \\
\left(s_{i} \rightarrow 1\right) & =1, \\
\left(s_{i} \rightarrow s_{j}\right) & =s_{k} \quad \text { if }\left(s_{i} \rightarrow s_{j}\right)=s_{k} \in\left\{s_{1}, \cdots, s_{n}\right\}, \\
& =1 \quad \text { otherwise, }
\end{aligned}
$$

where $i, j \in\{1, \cdots, n\}$.

This is a model of $P^{*}$, for firstly the axiom schemes are valid; if elements of $1, s_{1}, \cdots, s_{n}$ are substituted in to the axiom schemes the result is either 1 or a provable member of $s_{1}, \cdots, s_{n}$. Secondly, the rule preserves designation. If the conclusion is not designated, it is a nonprovable one of $s_{1}, \cdots, s_{n}$, but then the premise, containing the same propositional variables as the conclusion and of shorter length, is a member of $s_{1}, \cdots, s_{n}$. It cannot be a theorem (otherwise the conclusion would be) and so is not designated.

Finally $f$ is rejected in this model by the identity substitution for $f \in\left\{s_{1}, \cdots, s_{n}\right\}$ and is not provable.

\section{REFERENCES}

1. R. Harrop, On the existence of finite models and decision procedures for propositional calculi, Proc. Cambridge Philos. Soc. 54 (1958), 1-13.

2. - A relativisation procedure for propositional calculi with an application to a generalised form of Post's theorem, Proc. London Math. Soc. (3) 14(1964), 595-617.

University of New Brunswick, Canada 